\title{
Individual immune system responds differently.
}

\author{
Afridi S* and Azizullahathi
}

Centre for Advanced Drug Research, COMSATS Institute of Information Technology, Abbottabad, Pakistan

\begin{abstract}
Each individual immune system is highly specific but extremely distant from other individuals. Based on recent technological advancement whole immune system analysis reveal different immune profiling for every individual. Herein, we clarified the detailed variation in the human immune system with coherence to their respective effecting factors. The most influencing factors include age, time, gender, heritable, non-heritable, non-pathogenic, pathogenic microbes, genetic makeup and environment. Correlating these factors analysis will help us to investigate and treat numerous immune pathological conditions with highest specificity at the doorstep of every individual in the near future.
\end{abstract}

Keywords: Immune system, Immune system variations, Components variation, Recent technology, Factor affecting immune system.

Accepted on March 13, 2017

\section{Major Challenges with Immune System Analysis}

Ins Translating pre-clinical data into applied immunology was a true dream for immunologist. Reasonably due to lack of proper technology for human immune system analysis the acquired data from an animal model was not correlated oftenly. To investigate the status of the immune system directly, immunologists are now using new technologies. In this regard one of the best strategies is systems immunology or vaccinology. An extensive variety of variables can bother the human immune system, however the most advantageous to examine for frameworks immunology is the reaction to standard immunizations [1-3]. Systems vaccinology can uncover which parts of the insusceptible system change and how they change in light of perturbations and thus yield data about the sensitivities of a given individual's resistant immune system and the variety of safe reactions between people. By concentrating on blood, a frameworks immunology approach can be edifying about both health and disease, young and old persons. Over-all, systems immunology deal with the identification of key components of a given immune system and measure its components change in response to agitations of the system. Luckily, the dominant part of these components can be measured with accessible advance in technologies and a representation of these components is available in a blood test which is broadly accessible in human studies. Likewise, systems approaches make utilization of the way that specialized cells in the immune system are both the indicators and effectors of the immune system, that these cells communicate with each other through cytokines and direct associations and that a worldwide representation of what is going on in the safe arrangement of a specific individual at a given time can be evaluated by investigating such collaborations. In spite of the fact that blood does not constitute an immunological organ essentially, it is the channel for most resistant cells coursing in the body, particularly after an immunological stimulus, for example, vaccination $[4,5]$.

\section{High-tech advancement}

The current advancement of numerous new high throughput advances empowers concurrent estimations of numerous cell sorts, cytokines and different biomarkers of insusceptible capacity in a similar blood test by various techniques including FACS, ELISA, micro array, bead array, mass cytometry and sequencing etc. Briefly, FACS assay based on single cell analysis by fluorescently tag antibodies quantifications [6-8]. Micro array tell us about protein expression level and bead array based on fluorescent bead proteins readouts quenching. Similarly, mass cytometry are employed to single cell analysis using mass-reporters tag antibodies. Likewise another premature techniques formed by the combination of FACS-Mass cytometry are used to analyze $>120$ markers at the same time within a single cells. While, single cell sequencing give complete information about any target protein expression or mutations. Such advancement gives a chance to concentrate on human immune system variation on a universal scale, considering covariation of particular cell populations and protein contents $[9,10]$.

\section{Types of Immune System Variation}

Broadly, immune system variations can be divided into three main categories. The detailed of each category is given below.

\section{Variation within an individual}

In order to combat with day-to-day pathogen advancement, individual immune system is continuously changed. Normally a healthy immune system frequently adapts to its encounters with hostile pathogens, nutritional components, gut microbes, and more overwhelming the influences of the most heritable factors [11-13]. 


\section{Variation within interindividual}

It is broadly accepted that between individuals variations in immune systems exist. Yet the degree of variety is just beginning to distinctly clear. With the help of technology advancement, investigations empower assessments of interindividual variety, at the level of individual estimations as well as at the framework level across many people in a population. Data from immune cell recurrence estimations using mass cytometry revealed variation in the relative frequencies of primary insusceptible cell population; monocytes, natural killer (NK), B cells and CD4+ / CD8+ T cells. The recurrence of CD4+ T cells extends in the vicinity of $20-90 \%$ and the part of CD8 $+\mathrm{T}$ cells extends between $6-65 \%$. The B cell contribution was $4-69 \%$ within the aggregate of lymphocytes. The way that apparently healthy people show such an extended level of variety in particular immune system constituents recommends novel roads for future reviews into the instruments assuring strength and access to the immune system. The immune complex system adopts versatile arrangements, compensatory pathways and useful repetition to keep up its key capacities even in "anomaly" people [14].

\section{Variation within human population}

The composition of immune cells and related proteins within the immune system varies among different populations. It is imperative not to list just the scope of variety for individual estimations, but also to explore novel affiliations between immune system segments and the framework of variety between people. The structure of immune cells and proteins within an individual's immune system is known its immune type and it is intriguing to ponder whether such immune types are circulated as distinct groups or as a continuum [15-17]. Such a worldwide comprehension of the human immune system variety could assist to recognize people with exceptional immune types and immune type associated with expanded danger of extreme abnormalities and immunemediated diseases. Many scientists have used worldwide gene expression profiles to characterize variety between patients. For instance SLE is a disease infamous for its heterogeneous clinical introduction. However, in the light of worldwide gene expression profiles patients with SLE can be assembled into seven distinct groups of patients and disease seriousness [18].

\section{Factor Affecting Immune System Variation}

Recently it was shown that the human immune system vary with respect to age, gender and specific environment can be studied globally. Therefore a combined comprehension of both heritable and nonheritable influences on immunity is needed to fully understand interindividual variation and its possible outcomes on immune health and disease conditions. At his was reported that some key factors play a vital role in the development of a specific immune system (Figure 1). Beside other factors, i.e., occasional and occupational, these factors play a dynamic role in the development of a specific immune system. The detailed of some prominent factors are given bellow.

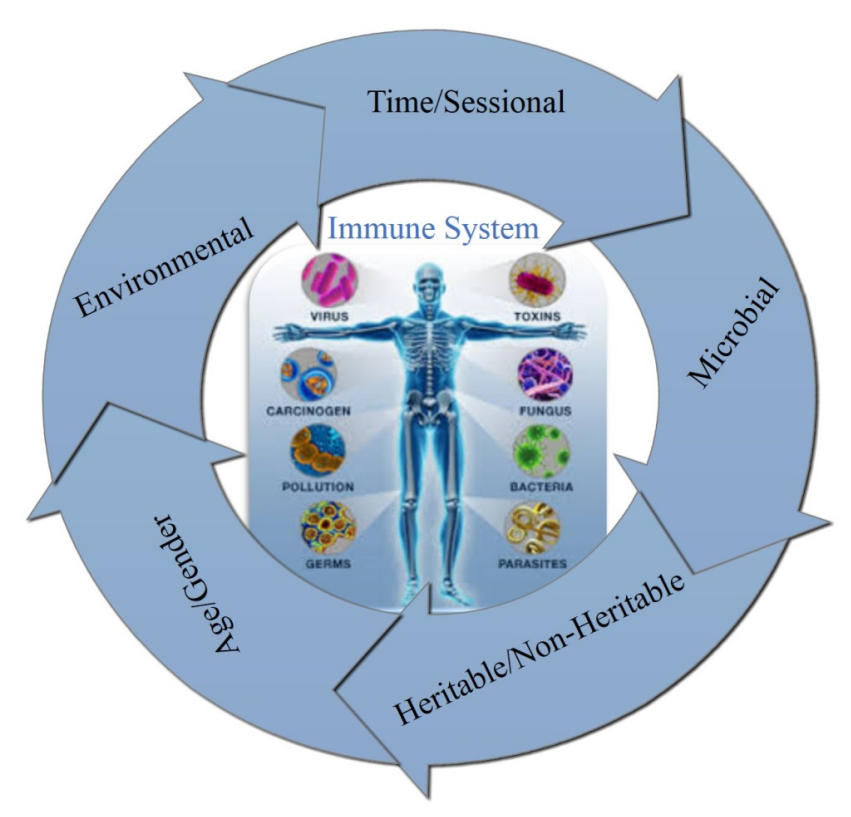

Figure 1. Human immune system development: Key factors that regulate human immune system growth, development and expansion.

\section{Age base variation}

Age base variation play vital role in immune system regulation, i.e., young children and aged people is more vulnerable to infections than other age groups [19,20]. Similarly, due to life in utero, the infant has an immune system swayed towards tolerance with predominantly naive phenotypes that mature when exposed to the environment. Compared to adults the neonatal immune system relies on discrete protective cell populations. On the other hand the immune systems of aged people respond slowly due to loss of immune cells, lymphopenia and decreased in diversity of variable receptor genes on lymphocytes $\mathrm{B}$ and $\mathrm{T}$ cells [21,22]. Low-grade inflammatory status of higher concentrations of proinflammatory cytokines (i.e., TNF) exist in aged people. About 24 protein biomarkers are highly influenced by the age factor $[23,24]$. However, it is important to note that even if certain factors are found to correlate positively or negatively with age. This cannot be taken as evidence of their contribution in the process of ageing. Environmental factors can often affect individuals differently during different stages of life and adaptive changes in the immune system to such aspects could explain age correlated immune system parameters [25].

\section{Time base variation}

Time base variation during an immune response seems to be an ongoing moving target within a specific individual. Nonetheless, this does not happen outside of such episodes, at least not in healthy adults. Analysis of such blood samples takes weeks to months apart from that healthy adults show highly stable immune cell frequencies and serum protein levels [26,27]. Recent studies over the course of annual samplings indicate that in healthy people immune profiles remain stable up to 6 years, suggesting that each individual makes a baseline state of well-regulated immune system framework. For example to an acute challenge the immune responses lead to 
extreme changes that involved a sharp increases in serum protein concentrations and immune cell populations, which return quickly to the same baseline state as before the challenge. All through the mechanisms that regulate such coordination are poorly understood, but still it becomes easier to study all system components within the same sample simultaneously with technology developments that will assist to improve our mechanistic understanding [28].

\section{Sessional variation}

Like other factor sessional variation also affects same or different individual immune system differently. By kids different type 1 diabetes change over the season, i.e., elevated in pre-winters and winter months with least occurrence in summer times generally in the northern half of the globe [29]. Similarly, numerous rheumatoid arthritis patients encounter periodic variations in joint indications due to alterations in immune cell structure and frequencies through the span of the year [30]. In line circadian, varies in fiery signs, i.e., rigidity and agony being most noticeably worse in morning, is a characterizing manifestation of rheumatoid arthritis. Suggesting the linkage of circadian direction toward the endogenous hormones cortisol that increase the intensity of interleukin-6 (IL-6) during morning hours [31,32].

\section{Gender base variation}

Comparatively women are more prone to various immunemediated abnormalities identified by $>80 \%$ patients with immune system disorders, i.e., SLE, Sjorgren syndrome and thyroid illness. However, the occurrence of ankylosis spondylitis is higher in men. While, concentrations of serum proteins and their functional immune responses properties between men and women are not clear. Similarly entire blood gene expression level examination demonstrated clear differences between men and women, both for autosomal and sex linked genes $[33,34]$. For example due to immunomodulatory effects of estrogen as an enhancer and testosterone as a suppressor of humoral immunity, it seems that women mount stronger immune responses than men. Recently it was found that gene expression level in man toward influenza virus vaccination was lower than women $[35,36]$.

\section{Heritable variation}

In many patients host genetics are deemed to be the most influential cause of infection. For instance, monogenic immunodeficiency manifestation will be more severe in secondary infections which lead to a greater extent of complex genetic predisposition. To explore the possible heritable traits that affect the specific immune system measurements, various studies have been initiated, i.e., Genome Wide Association Studies (GWAS) represent the affiliation of genetic loci with individual immune system measurements including specific cytokine or immune cells concentration and frequencies [37]. Similarly, GWAS also deal in with the finding of individual immune system measurements with the specific genetic loci that are responsible for the disease prevalence specially the autoimmune conditions (genetic risk variants $>80$ ) which have been widely studied over the past and affecting humans in many ways. Therefore, more considerations have been placed on the heritable factors that influence the immune system components including immune cells frequencies and serum protein concentration, in the current scenario while some of the studies performed on immune cell functions give analysis of the discern heritable influences [38].

Immune cells frequencies: During acute infections white blood cell (WBC) count increased with a moderate heritability of 0.38 ( 0.14 basophils; 0.4 monocytes) and the reason to the inter-individual variations could be the specific loci, i.e., chromosome 2 loci are associated with coeliac disease and monocyte count $[39,40]$. Similarly with the help of high dimensional Flow Cytometry approach about 24 additional loci that affect $>20$ immune cells populations were identified $[41,42]$.

Serum proteins profile: Dysregulated serum proteins (cytokines) profiles relate to several immune mediated disorders. Strong correlation exists between cytokine genes and immune mediated diseases, i.e., SLE are dominated by interferon-inducible gene within the blood however, the pathological influence of such genetic associations is already difficult to understand [43]. Recent inter-individual variations studies on APC and T-cells results estimated about $22 \%$ variation as genetically heritable factors and suggest its moderate contribution to affect blood transcriptomes [44]. All these information gave us an estimation of variations among the individuals within a population.

\section{Non-heritable variation}

To maintain their efficiency over time immune system serves as a sensory and regulatory system for external or internal stimuli $[45,46]$. Suggesting that these stimuli can alter the composition and functional property of an individual's immune system. These non-heritable factors are typically, infections and vaccines, as well as de novo mutations and stochastic epigenetic variations, in addition to pathogenic and symbiotic microorganism influence. Stochastic epigenetic changes occur during an immune cells division and influence immune cell phenotypes [47].

\section{Microbial base variation}

The development of the immune system also depends on the encounter of microorganisms' types and their threshold, i.e., GIT bacterial strains (E. coli) interaction with lymphoid tissues promotes their normal development, while in germ free animal functional deficiencies are observed, suggest the co-evolution. The controlled animal facility environment has positive as well as negative effects on the study of the relationship between microbiota and immune system. On the other hand microbiota in human GIT has been associated with inflammation and diseases complications like Ulcerative Colitis and Crohn Diseases [48,49]. Similarly, humoral response between microorganisms and non-adjuvant vaccines, i.e., bacterial flagellin sensors as a vaccines stimulus is responsible for plasma cell activation and antibody production [50]. Therefore, differences in gut microbiota may depict different responses in 
specific individuals. Likewise, many viruses became the cause of chronic disease while some are integrated into our genome, i.e., cytomegalovirus (CMV) acts as modulators for human immune systems. About 10\% CMV specific T cell and NK cells change their phenotypes to CMV stimulus. CMV analysis within 20-30 year age groups was associated with beneficial flu vaccine mediated immune response in immune compromised people. Constant infection with low grade virulence viruses induces immune response and promotes immune mediated pathologies [51-53].

\section{Environmental factors}

Environmental factors like other units deeply affect individual immune system development and regulation. Beside microorganisms, many environmental factors influenced the development and reshaping our immune system. One of the most harmful factors is the cigarette smoking along with its $>4000$ components which damage human's lungs and systemic system that result in increased leukocyte count and reduced serum immunoglobulins and NK-cells activity [54,55]. Similarly Smoking further leads to altered antibody specificity that produces modified peptides, i.e., citrullinated peptides, which have a clinical importance in various autoimmune diseases including rheumatoid arthritis [56,57]. Furthermore, industrial west, heavy metals, vehicle smoke, plastic fire, acid rain and animal debris etc. also affect badly immune system development [58-60].

\section{Conclusion}

With the help of available analytical techniques, we can easily differentiate inter-individuals immune system components variations in both healthy and diseased cases. These analysis techniques will allow us to predict the onset of disease threshold and it's curing immunological parameters. Similarly, personalized immune therapies (with focused immune cells vary) for cancer and related immune pathological conditions would help us to interpret the main cause of variations. In broad spectrum, the knowledge about individual's stable and functional immune system state will help us to provide better and long lasting health care for the whole population through alteration modifiable factors conditions. Briefly, host genetics, age, sex, environment, heritable and non-heritable factors are the influential cause of infection in many patients. However more considerations have been given to the heritable factors that influence the components of individual immune system, i.e., population of immune cells and serum protein concentration. Overall, in the development and progression of individual immune system specifications these factors play a significant role coherently.

\section{References}

1. Hayday AC, Peakman M. The habitual, diverse and surmountable obstacles to human immunology research. Nature immunology. 2008; 9: 575-580.

2. Hagan T, Nakaya HI, Subramaniam S, et al. Systems vaccinology: Enabling rational vaccine design with systems biological approaches. Vaccine. 2015; 33: 5294-5301.

3. Davis MM. A prescription for human immunology. Immunity. 2008; 29: 835-838.

4. Steinman RM, Mellman I. Immunotherapy: bewitched, bothered, and bewildered no more. Science. 2004; 305: 197-200.

5. Afridi S, Hoessli DC, Hameed MW. Mechanistic understanding and significance of small peptides interaction with MHC class II molecules for therapeutic applications. Immunological reviews. 2016; 272: 151-168.

6. Afridi S. A cyclic peptide accelerates the loading of peptide antigens in major histocompatibility complex class II molecules. Biochemical and biophysical research communications. 2015; 456: 774-779.

7. Bendall SC. Single-cell mass cytometry of differential immune and drug responses across a human hematopoietic continuum. Science. 2011; 332: 687-696.

8. Delehanty JB, Ligler FS. A microarray immunoassay for simultaneous detection of proteins and bacteria. Analytical Chemistry. 2002; 74: 5681-5687.

9. Newell EW, Sigal N, Bendall SC, et al. Cytometry by time-of-flight shows combinatorial cytokine expression and virus-specific cell niches within a continuum of CD8+ T cell phenotypes. Immunity. 2012; 36: 142-152.

10. Chattopadhyay P, Roederer M. A mine is a terrible thing to waste: High content, single cell technologies for comprehensive immune analysis. American Journal of Transplantation. 2015; 15: 1155-1161.

11. Schmid-Hempel P. Variation in immune defence as a question of evolutionary ecology. Proceedings of the Royal Society of London B: Biological Sciences. 2003; 270: 357-366.

12. Costello EK, Lauber CL, Hamady M, et al. Bacterial community variation in human body habitats across space and time. Science. 2009; 326: 1694-1697.

13. Clemente JC, Ursell LK, Parfrey LW, et al. The impact of the gut microbiota on human health: an integrative view. Cell. 2012; 148: 1258-1270.

14. Brodin P. Variation in the human immune system is largely driven by non-heritable influences. Cell. 2015; 160: 37-47.

15. Banchereau R. Personalized immunomonitoring uncovers molecular networks that stratify lupus patients. Cell. 2016; 165: 551-565.

16. Bhattacharya S. ImmPort: disseminating data to the public for the future of immunology. Immunologic research. 2014; 58: 234-239.

17. Whitney AR. Individuality and variation in gene expression patterns in human blood. Proceedings of the National Academy of Sciences. 2003; 100: 1896-1901.

18. Casanova J-L, Abel L. The human model: a genetic dissection of immunity to infection in natural conditions. Nature Reviews Immunology. 2004; 4: 55-66.

19. Dowling DJ, Levy O. Ontogeny of early life immunity. Trends in immunology. 2014; 35: 299-310. 
20. Simon AK, Hollander GA, McMichael A. Evolution of the immune system in humans from infancy to old age. The Royal Society. 2015.

21. Goronzy JJ, Weyand CM. Ageing, autoimmunity and arthritis: T-cell senescence and contraction of T-cell repertoire diversity-catalysts of autoimmunity and chronic inflammation. Arthritis Res Ther. 2003; 5: 225.

22. Qi Q. Diversity and clonal selection in the human T-cell repertoire. Proceedings of the National Academy of Sciences. 2014;111:13139-13144.

23. Michaud M. Proinflammatory cytokines, aging, and agerelated diseases. Journal of the American Medical Directors Association. 2013; 14: 877-882.

24. Enroth S, Johansson Å, Enroth SB, et al. Strong effects of genetic and lifestyle factors on biomarker variation and use of personalized cutoffs. Nature communications. 2014; 5 .

25. Sørensen TI, Nielsen GG, Andersen PK, et al. Genetic and environmental influences on premature death in adult adoptees. New England Journal of Medicine. 1988; 318: 727-732.

26. Tsang JS. Global analyses of human immune variation reveal baseline predictors of postvaccination responses. Cell. 2014; 157: 499-513.

27. Carr EJ. The cellular composition of the human immune system is shaped by age and cohabitation. Nature immunology. 2016.

28. Gibbons DL. Neonates harbour highly active $\gamma \delta \mathrm{T}$ cells with selective impairments in preterm infants. European journal of immunology. 2009; 39: 1794-1806.

29. Moltchanova E, Schreier N, Lammi N, et al. Seasonal variation of diagnosis of Type 1 diabetes mellitus in children worldwide. Diabetic Medicine. 2009; 26: 673-678.

30. Dopico XC. Widespread seasonal gene expression reveals annual differences in human immunity and physiology. Nature communications. 2015; 6.

31. Straub RH, Cutolo M. Circadian rhythms in rheumatoid arthritis: implications for pathophysiology and therapeutic management. Arthritis \& Rheumatology. 2007; 56: 399-408.

32. Cutolo M, Straub RH. Circadian rhythms in arthritis: Hormonal effects on the immune/inflammatory reaction. Autoimmunity reviews. 2008; 7: 223-228.

33. Lee W, Reveille JD, Davis JC, et al. Are there gender differences in severity of ankylosing spondylitis? Results from the PSOAS cohort. Annals of the rheumatic diseases. 2007; 66: 633-638.

34. Pilia G. Heritability of cardiovascular and personality traits in 6,148 Sardinians. PLoS Genet. 2006; 2: e132.

35. Cutolo M. Sex hormones influence on the immune system: basic and clinical aspects in autoimmunity. Lupus. 2004; 13: 635-638.

36. Furman D. Systems analysis of sex differences reveals an immunosuppressive role for testosterone in the response to influenza vaccination. Proceedings of the National Academy of Sciences. 2014; 111: 869-874.
37. Kochi Y. Genetics of autoimmune diseases: Perspectives from genome-wide association studies. International immunology. 2016; 28: 155-161.

38. Gregersen PK, Olsson LM. Recent advances in the genetics of autoimmune disease. Annual review of immunology. 2009; 27: 363-391.

39. Nalls MA. Multiple loci are associated with white blood cell phenotypes. PLoS Genet. 2011; 7: e1002113.

40. Garner C, Murray J, Ding Y, et al. Replication of celiac disease UK genome-wide association study results in a US population. Human molecular genetics. 2009; 18: 4219-4225.

41. Orrù V. Genetic variants regulating immune cell levels in health and disease. Cell. 2013; 155: 242-256.

42. Roederer M. The genetic architecture of the human immune system: a bioresource for autoimmunity and disease pathogenesis. Cell. 2015; 161: 387-403.

43. Ollier WE. Cytokine genes and disease susceptibility. Cytokine. 2004; 28: 174-178.

44. De Jager PL, Hacohen N, Mathis D, Regev A, Stranger $\mathrm{BE}$, Benoist $\mathrm{C}$, eds. ImmVar project: Insights and design considerations for future studies of "healthy" immune variation. 2015.

45. Grossman Z, Paul WE. Adaptive cellular interactions in the immune system: the tunable activation threshold and the significance of subthreshold responses. Proceedings of the National Academy of Sciences. 1992; 89: 10365-10369.

46. Brodin P, Kärre K, Höglund P. NK cell education: not an on-off switch but a tunable rheostat. Trends in immunology. 2009; 30: 143-149.

47. Fraga MF. Epigenetic differences arise during the lifetime of monozygotic twins. Proceedings of the National Academy of Sciences of the United States of America. 2005; 102: 10604-10609.

48. Dalal SR, Chang EB. The microbial basis of inflammatory bowel diseases. The Journal of clinical investigation. 2014; 124: 4190-4196.

49. Alcaïs A, Quintana-Murci L, Thaler DS, et al. Lifethreatening infectious diseases of childhood: single-gene inborn errors of immunity? Annals of the New York Academy of Sciences. 2010; 1214: 18-33.

50. Oh JZ. TLR5-mediated sensing of gut microbiota is necessary for antibody responses to seasonal influenza vaccination. Immunity. 2014; 41: 478-492.

51. Furman D. Cytomegalovirus infection enhances the immune response to influenza. Science translational medicine. 2015; 7: 281ra243-281ra243.

52. Xu GJ. Comprehensive serological profiling of human populations using a synthetic human virome. Science. 2015; 348: aaa0698.

53. Sylwester AW. Broadly targeted human cytomegalovirusspecific CD4+ and CD8+ T cells dominate the memory compartments of exposed subjects. Journal of Experimental Medicine. 2005; 202: 673-685.

54. Sopori M. Effects of cigarette smoke on the immune system. Nature Reviews Immunology. 2002; 2: 372-377. 
55. Ferson M, Edwards A, Lind A, et al. Low natural killercell activity and immunoglobulin levels associated with smoking in human subjects. International Journal of Cancer. 1979; 23: 603-609.

56. Mathews J, Whittingham S, Hooper B, et al. Association of autoantibodies with smoking, cardiovascular morbidity, and death in the Busselton population. The Lancet. 1973; 302: 754-758.

57. Padyukov L, Silva C, Stolt P, et al. A gene-environment interaction between smoking and shared epitope genes in HLA-DR provides a high risk of seropositive rheumatoid arthritis. Arthritis \& Rheumatology. 2004; 50: 3085-3092.

58. Anwar A, Shah MR, Muhammad SP, et al. Thiopyridinium capped silver nanoparticle based supramolecular recognition of $\mathrm{Cu}$ (I) in real samples and T-lymphocytes. New Journal of Chemistry. 2016; 40: 6480-6486.

59. Khan B. Synthesis, characterization and $\mathrm{Cu} 2+$ triggered selective fluorescence quenching of Bis-calix [4] arene tetra-triazole macrocycle. Journal of hazardous materials. 2016; 309: 97-106.

60. Rasheed W, Shah MR, Kazmi MH, et al. Sterically stabilized fluorescent silver nanoconjugates for optical discrimination of $\mathrm{Cu}$ (ii) in real samples and in vitro bioimaging of the $\mathrm{Cu}$ (ii) scavenging process in human lymphocytes by atomic force microscopy. New Journal of Chemistry. 2016; 40: 5546-5554.

\section{*Correspondence to}

Afridi $S$

Centre for Advanced Drug Research

COMSATS Institute of Information Technology

Abbottabad, 22060, Pakistan 